\title{
Effect of Solvents on Size and Morphologies Of sno Nanoparticles via Chemical Co-precipitation Method
}

\author{
G.Vijayaprasath ${ }^{1}$ \\ Department of physics, \\ Alagappa University, \\ Karaikudi - 630 004, India.
}

\author{
G.Ravi $^{1}$ \\ Department of physics, \\ Alagappa University, \\ Karaikudi - 630 004, India.
}

\author{
Y.Hayakawa ${ }^{2}$ \\ Shizuoka University, \\ Hamamatsu - 432 8011, \\ Japan
}

\begin{abstract}
Stannous oxide ( $\mathrm{SnO})$ is an important functional material which contributes to a wide range of applications. In present study, the $\mathrm{SnO}$ nanoparticles were synthesized using different solvents by chemical co-precipitation method. Different morphologies of $\mathrm{SnO}$ nanoparticles have been obtained by different solvents. The size of $\mathrm{SnO}$ nanoparticles was estimated by powder X-ray diffraction (PXRD) pattern and revealed that $\mathrm{SnO}$ crystallizes into tetragonal rutile phase. The chemical structural information of the synthesized nanoparticles was studied by Fourier transform infrared (FTIR) spectroscopy. The prominent UV emission peak was observed at $358 \mathrm{~nm}$ in the luminance spectra, studied by photoluminescence (PL) spectroscopy. The composition of the product SnO nanoparticles could be determined by X-ray photoelectron spectroscopy (XPS) and the binding energies of O $1 \mathrm{~s}$ and $\mathrm{Sn} 3 \mathrm{~d}_{3 / 2}$ have been found which are centered at 530 and $495 \mathrm{eV}$, respectively.
\end{abstract}

Keywords: Stannous oxide; semiconductor; nanoparticles; morphologies; co-precipitation

\section{INTRODUCTION}

Nanostructured materials have attracted great interest in both fundamental as well as applied research areas due to their outstanding physical and chemical properties and also promising applications in nanodevices [1]. A reduction in particle size to nanometer scale results in various interesting properties compared to the bulk properties. Stannous oxide $(\mathrm{SnO})$ is one of the best p-type semiconductors found in literature for various applications. The naturally formed tin $(\mathrm{Sn})$ vacancies causes the variable direct band gap in $\mathrm{SnO}$, has attracted considerable attention owing to its specific functional characteristics and significance in a various technological applications such as coating substrate, catalyst, thin film transistors, etc.[2]. Variety of solution phase techniques like homogeneous co-precipitation [3], hydrothermal route [4], sol gel method [5], sol gel combustion [6], mechanical ball milling [7], and sonochemical method [8] have been employed to synthesis metal oxides. Among these, co-precipitation method is a simple and less expensive and requires simple instrumentation. A variety of $\mathrm{SnO}$ distinct morphologies have been successfully synthesized such as particle, platelet [9], flower [10], whiskers [11] and dendrites [12]. However, the implementation of nanostructure's preparation of $\mathrm{SnO}$ with well controlled size and morphology by chemical co-precipitation method is a great challenge for nanomaterials synthesis. Here, we report the size and morphological changes of $\mathrm{SnO}$ nanoparticles using different solvents. The properties of as-synthesized $\mathrm{SnO}$ nanoparticles were determined by using XRD, FTIR, SEM, PL and XPS.

\section{EXPERIMENTAL DETAILS}

In the synthesis process, all reagents of analytical grade (AR) were used without further purification. $0.36 \mathrm{M}$ of tin chloride $\left(\mathrm{SnCl}_{2}\right)$ was dissolved in different solvents such as de-ionized water, ethanol, ethylene glycol and propanol. Then, $\mathrm{NaOH}$ solution was added drop wise into the solution to maintain the $\mathrm{pH}$ of solution between 8-11. The resultant solution was stirred constantly for 60 minutes and then after vigorous stirring, white suspension was obtained. The product was centrifuged, filtered out and rinsed with alcohol and deionized water several times to remove soluble impurities if any in the solution, and then dried at $60^{\circ} \mathrm{C}$ for $1 \mathrm{~h}$ in air atmosphere, and finally the product was collected for characterization.

\section{RESULTS AND DISCUSSION}

\subsection{Structural Analysis}

The PXRD patterns of synthesized SnO nanoparticles using different solvents are shown in figure1. The diffraction peaks are well matched with the standard SnO reflections (JCPDS Card No. 06 - 0395).

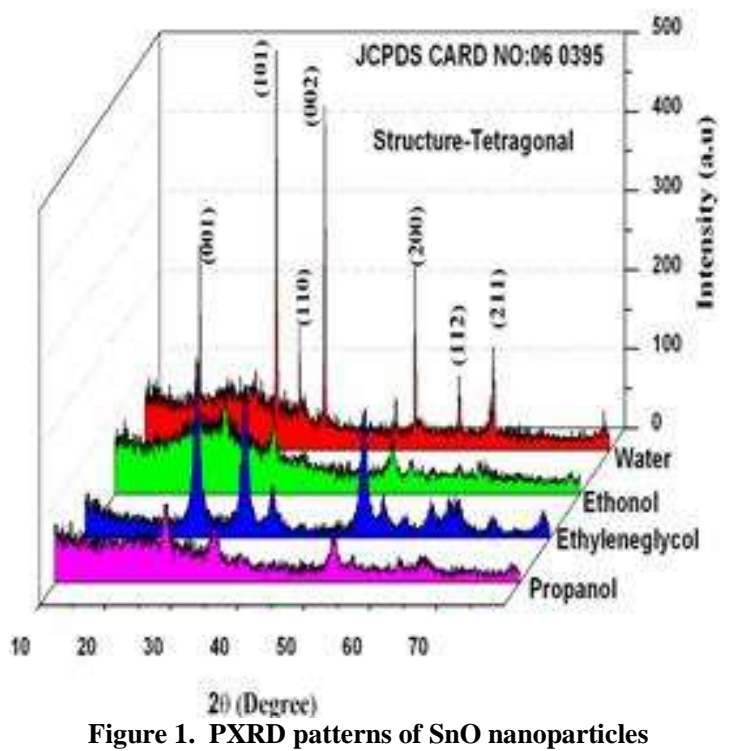

The X-ray pattern reveals that $\mathrm{SnO}$ nanoparticles are having tetragonal wurtzite structure and the obtained peaks are indexed to (001), (101), (110), (002), (200), (112) and (211) planes. The reduction in peak intensities and broad FWHM were observed for the samples synthesized in solvents of ethanol and propanol. The calculated values of grain size, dislocation density and strain are tabulated in table 1 . 
Table 1. Grain size and micro structural parameters of SnO nanoparticles

\begin{tabular}{|c|l|c|c|c|}
\hline $\begin{array}{c}\text { Sl. } \\
\text { No }\end{array}$ & Solvent & $\begin{array}{c}\text { Grain } \\
\text { Size } \\
\mathbf{D = 0 . 9} \lambda / \\
\boldsymbol{\beta c o s} \boldsymbol{\theta} \\
\mathbf{( n m )}\end{array}$ & $\begin{array}{c}\text { Dislocation } \\
\text { Density } \\
\boldsymbol{\delta}=\mathbf{1} / \mathbf{D}^{2} \\
\left(\mathbf{l i n e s} / \mathbf{m}^{\mathbf{2}}\right)\end{array}$ & $\begin{array}{c}\text { Micro } \\
\text { Strain } \\
\boldsymbol{\varepsilon}=\boldsymbol{\beta} \\
\mathbf{c o s} \boldsymbol{\theta} / \mathbf{4} \\
(\mathbf{l i n e s} \\
\left.\mathbf{2} / \mathbf{m}^{\mathbf{4}}\right)\end{array}$ \\
\hline 1. & Water & 40.83 & $0.599 \mathrm{E}+15$ & $0.849 \mathrm{E}-03$ \\
\hline 2. & Ethanol & 13.61 & $5.3982 \mathrm{E}+15$ & $2.547 \mathrm{E}-03$ \\
\hline 3. & Ethylene & 8.17 & $14.980 \mathrm{E}+15$ & $4.243 \mathrm{E}-03$ \\
\hline 4. & Propanol & 6.81 & $21.584 \mathrm{E}+15$ & $5.093 \mathrm{E}-03$ \\
\hline
\end{tabular}

\subsection{Functional Analysis}

FTIR spectrum is a useful tool to understand the functional groups of any organic molecule. The FTIR spectra of $\mathrm{SnO}$ nanoparticles collected using different solvents are shown in Figure 2. Metal oxides generally give absorption band below $1000 \mathrm{~cm}^{-1}$ arising from inter-atomic vibrations.

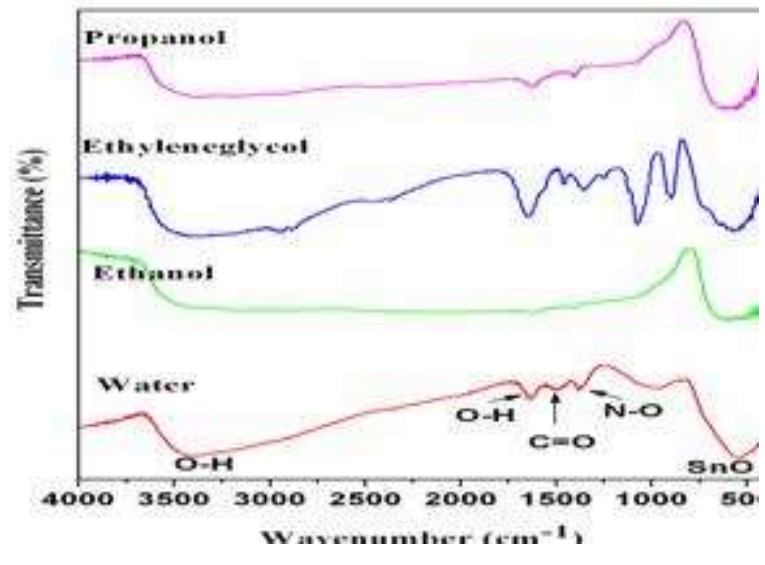

Figure 2. FTIR spectra of SnO nanoparticles

For all synthesized products, the absorption peaks are found at 3456 and $1618 \mathrm{~cm}^{-1}$ are attributed mainly to the $\mathrm{O}-\mathrm{H}$ stretching vibration of surface hydroxyl group or adsorbed water of the $\mathrm{SnO}$ nanoparticles [13]. The principal peak observed at $1586 \mathrm{~cm}^{-1}$ corresponds to the strong asymmetric stretching of $\mathrm{C}=\mathrm{O}$ bond. The presence of $\mathrm{N}-\mathrm{O}$ is conformed from the peak observed at $1409 \mathrm{~cm}^{-1}$. The absorption band at $515 \mathrm{~cm}^{-1}$ is ascribed to the terminal oxygen vibration of $\mathrm{SnO}$ [14].

\subsection{Morphological Analysis}

Figure 3 shows the SEM micrograph of $\mathrm{SnO}$ using different solvents. The formation of plate like morphology is obtained using water as a solvent and it produces better morphology than the other solvents like ethanol, ethylene glycol and propanol. The formation of $\mathrm{SnO}$ nanoparticles are highly agglomerated for other solvents because of the formation of smaller particles. But for the case of water solvent, the size becomes higher and it produces less agglomerated particles.

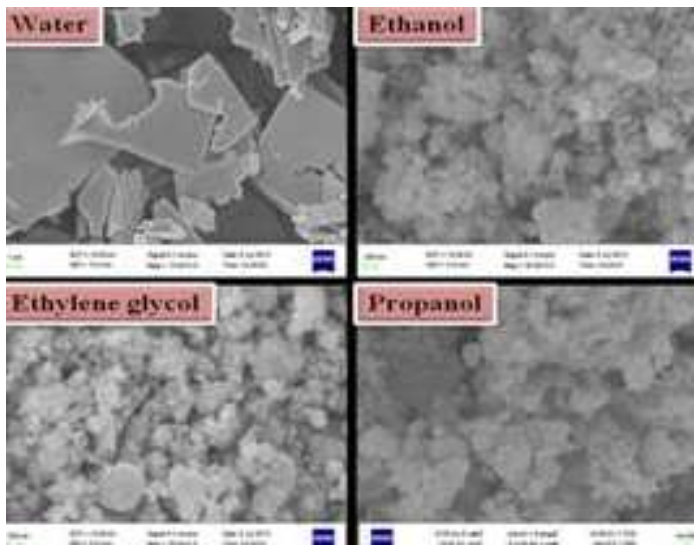

Figure 3. SEM images of SnO nanoparticles using different solvents

\subsection{Photoluminescence studies}

Figure 4 shows the room-temperature photoluminescence spectra collected by using an excitation wavelength of 325 $\mathrm{nm}$. A strong UV emission peak located at $358 \mathrm{~nm}(3.4 \mathrm{eV})$ corresponding to the near band-edge emission observed for all samples. The propanol mediated sample gives a very prominent UV emission. However, ethanol, ethylene glycol and water mediated samples show less intense UV emission peak comparable with the propanol mediated sample. HuanMing Xiong et al reported the surface state is regarded as a main factor that determines $\mathrm{SnO}$ visible luminescence, because $\mathrm{SnO}$ visible emission arises from its defects or vacancies which mainly located on the nanoparticles surface [15].

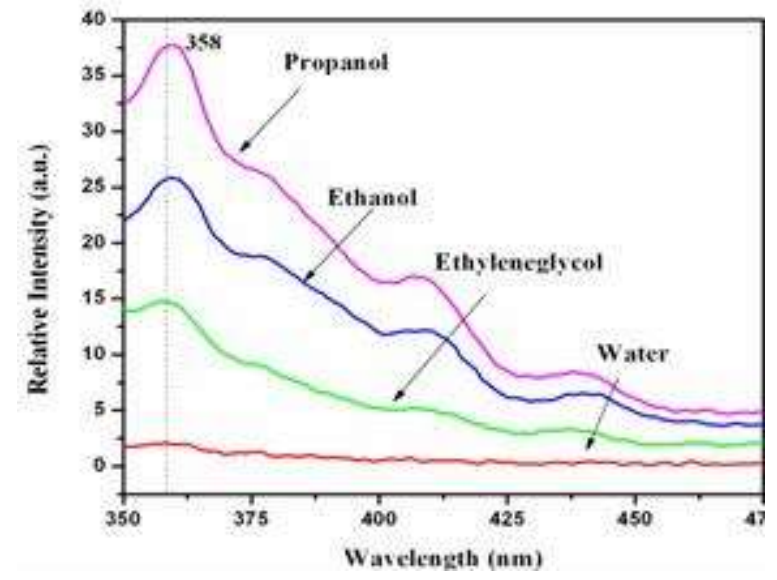

Figure 4. PL spectra of SnO nanoparticles

\subsection{Compositional Analysis}

Figure 5 shows the high resolution XPS spectra of assynthesized nanoparticles. The dominant signals corresponding to $\mathrm{Sn} 3 \mathrm{~d}_{5 / 2}, \mathrm{Sn}_{3} \mathrm{~d}_{3 / 2}$ and $\mathrm{O} 1 \mathrm{~s}$ are found at 486.2, 495.4 and $530.7 \mathrm{eV}$ respectively. These values are matching well with the previous reports for $\mathrm{SnO}$ [16]. The O1s photoelectron peak is observed at $530.7 \mathrm{eV}$ revealed that oxygen atoms are bonded to the Sn atoms. 

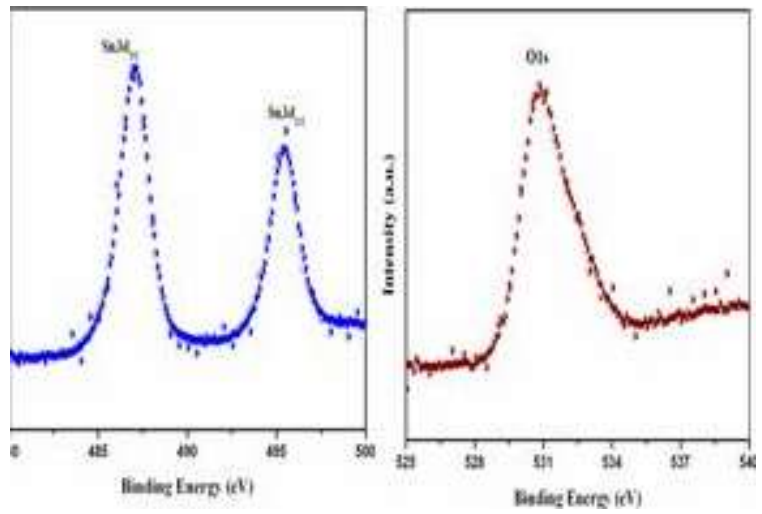

Figure 5. XPS spectra of SnO nanoparticles

\section{CONCLUSION}

Controllable size and different morphologies of $\mathrm{SnO}$ nanoparticles have been synthesised using different solvents by co-precipitation method. The size of the $\mathrm{SnO}$ nanoparticles has been estimated by PXRD. The chemical structural information of the product $\mathrm{SnO}$ has been studied by FTIR spectra. The different solvents give various morphologies studied by SEM analysis. The intensity of UV emission peaks are found to be varied with respect to the solvents used. The composition of the $\mathrm{SnO}$ nanoparticles has been determined by $\mathrm{X}$-ray photoelectron spectroscopy (XPS).

\section{REFERENCES}

[1] Kuang, D.B., Lei, B.X., Pan, YP., Yu, X.Y., Su, C.Y., J Phys Chem C., 113,(2009), 5508-5513.

[2] Zubair, M. Iqbal,Fengping Wang,Ting Feng,MRS Bull.,47,(2012),3902-3907.

[3] Yang, X., Song, H., Zhang, X., Ao, W., Qiu, G.Mater.Lett.57,(2003),3124-3127

[4] CChiu, H., SYeh, C., .J.Phys.Chem.C.111,(2007),72567259 .

[5] Gu, F., Wang, S.F., Lu, M.K., .Zhou, G.J., Xu, D.D., Yuan., R.J., Phys.Chem.B,108,(2004), 8119-8123.

[6] Fraigi, L.,. Lamas, D.G., Walsoe, N.E., de Reca, Nanostruct.Mater.11,(1999),311-318.

[7] Li, H., Huang, X.J., Chen, L., J.Power sources,81,(1999), 340-345.

[8] Majumdar, S., Chakraborty, S.E. Devi, P.S., A.Sen, Mater.Lett.,62,(2008),1249-1251.
[9] Krishnakumar, T., Pinna, N. Kumari, K.P., Perumal, K. Mater.Lett. 62, (2008), 3437-3440.

[10] Iqbal, M.Z., Wang, F.P., Javed, Q., Rafique, M.Y. Qiu , G.Nabi, H.M., Mater.Lett.,75, (2012), 236-239.

[11] Jia, Z.J., Zhu, L.P., Liao, G.H., Yu, Y., Tang, Y.W., Solid state Commun, 132,(2004),79-82.

[12] Orlandi, M.O., ELeite, .R., J.Phy.Chem.B, 110, (2006), 6621-6625.

[13] Masoud Salavati-Niasari, Noshin Mir, Fatemeh Davar, Inorganica Chimica Acta,363, (2010), 1719-1726.

[14] .Mariammal, R.N., Rajamanickam, N., Ramachandra, K., J.Nano-Electron. Phys. 3,(2011), 92-100.

[15] Huan-Ming Xiong, J. Mater. Chem., 20 (2010), 4251

[16] Kwoka,M., Waczynska, N., Koscielniak, P. Sitarz,J,Szuber, M. Thin Solid Films, 520,(2011), 913917. 